\title{
Vom Experiment zur Praxis: Wie moralische Argumente wirtschaftliche Selbstinteressen beeinflussen
}

\author{
Martin Schröder
}

\author{
MPIfG Journal Article \\ Martin Georg Schröder: Vom Experiment zur Praxis: Wie moralische Argumente wirtschaftliche Selbstinteressen beeinflussen. \\ In: Kölner Zeitschrift für Soziologie und Sozialpsychologie 63(1), 61 - 81 (2011). Springer VS \\ The original publication is available at the publisher's web site: http://dx.doi.org/10.1007/s11577-011-0129-2
}

\begin{abstract}
Zusammenfassung: Wie beeinflussen moralische Argumente wirtschaftlich selbstinteressiertes Handeln? Laborexperimente von Verhaltensökonomen untersuchen dies, doch ihre zentralen Ergebnisse wurden noch nicht in der Praxis getestet. Ein Forschungsprojekt rekonstruierte anhand von Fallstudien, wie moralische Argumente in Diskussionen um Produktionsverlagerung wirtschaftliche Selbstinteressen beeinflussen. Es zeigte sich, dass moralisches Verhalten, welches im Diktator- Ultimatum- und Kollektivgutspiel nachgewiesen wird, auch reales wirtschaftliches Handeln beeinflusst. Menschen verzichten auf die Maximierung ihres materiellen Nutzens, um moralischen Verhaltensregeln zu entsprechen. Statt strikter materieller Nutzenmaximierung verhalten sie sich reziprozitär. Die Ergebnisse verhaltensökonomischer Experimente, aber auch die Rekonstruktion wirtschaftlichen Handelns, verweisen somit auf ein Akteursmodell, das Gegenseitigkeit und auch gesellschaftliche Rolle beachtet und dadurch wirtschaftliches Handeln in vielen Situationen präziser erklärt als bisher vorherrschende Modelle. Dieses Modell wiederum in Laborexperimenten zu testen, wird aufgrund der Ergebnisse dieses Artikels nahegelegt.
\end{abstract}

Schlüsselwörter: Verhaltensökonomie · Empirische Wirtschaftsforschung · Experimentelle Wirtschaftsforschung $\cdot$ Moral $\cdot$ Reziprozität

\section{From behavioral experiments to case studies: how moral arguments influence economic self-interest}

\begin{abstract}
How do moral arguments influence economic self-interests? Behavioral economists try to answer this question using experiments, but their central results have not yet been tested in real economic life. A research project therefore reconstructed how moral arguments influenced economic self-interests in case studies of discussions about relocation. The results indicate that an
\end{abstract}

(C) VS Verlag für Sozialwissenschaften 2011

Dieses Forschungsprojekt lief am Max-Planck-Institut für Gesellschaftsforschung und wäre ohne dessen hervorragende Unterstützung unmöglich gewesen. Besonders bedanken möchte sich der Autor bei Wolfgang Streeck sowie bei Sigrid Quack, Guido Möllering und Jan Sauermann für hilfreiche Hinweise. Die Verantwortung für mögliche Fehler und alle Aussagen liegt allein beim Autor. Ein Buch, in dem die Fallstudien ausführlicher besprochen werden, erscheint 2011.

M. Schröder $(\bowtie)$

Max-Planck-Institut für Gesellschaftsforschung,

Paulstr. 3, 50676 Köln, Deutschland

E-Mail: schroeder@mpifg.de 
orientation towards morality, as in the dictator- ultimatum- and collective goods game, also seems to influence how interests are defined and pursued in real economic life. Instead of maximizing economic self-interest, actors behave reciprocally. Results of behavioral experiments as well as the observation of case studies of economic behavior therefore hint towards economic actors that are influenced by reciprocity and social roles. This allows for a more precise understanding of economic behavior in many situations. Testing this in experiments of behavioral economics is in turn advocated based on the results of this article.

Keywords: Behavioral economics $\cdot$ Empirical economic-research $\cdot$ Experimental economic-research $\cdot$ Morality $\cdot$ Reciprocity

\section{Was Soziologen und Verhaltensökonomen voneinander lernen können}

Experimente von Verhaltensökonomen zeigen, dass menschliches Handeln nicht dem neoklassischen Modell eines Akteurs entspricht, der ausschließlich seinen materiellen Nutzen maximieren will. Stattdessen beeinflussen auch Vertrauen, Moral- und Reziprozitätsüberlegungen menschliches Handeln. Doch es gibt Kritik an den Experimenten, die zu diesen Resultaten führen: „Man mag nun einwenden, dass solche einfachen Vertrauensspiele, die oft unter anonymen Bedingungen und mit nur einmaliger Interaktion untersucht werden, zu abstrakt sind, um der ,komplexen Realität' und dem ,komplexen Vertrauensphänomen' gerecht zu werden“ (Greiner und Ockenfels 2009, S. 222). Es stellt sich somit die Frage, inwiefern auch in der wirtschaftlichen Realität, außerhalb der Labore, in denen Verhaltensökonomen ihre Forschung betreiben, Handeln durch moralische Argumente, Vertrauen und Reziprozitätsüberlegungen beeinflusst ist. Beeinflussen moralische Argumente des jeweiligen sozialen Umfelds beispielsweise unternehmerische Strategien? Dies untersuchte ein Forschungsprojekt durch die Analyse von Produktionsverlagerungsdiskussionen. Dieser Artikel präsentiert dessen wichtigste Ergebnisse. In einem ersten Schritt beschreibt er, inwieweit typische Experimente und Ergebnisse der Verhaltensökonomie zeigen, dass menschliches Verhalten moralisch beeinflusst wird. Anhand dessen, was in Diskussionen um Produktionsverlagerung geschah, zeigt er daraufhin, inwiefern die durch Verhaltensökonomen herausgearbeiteten Mechanismen moralischer Beeinflussung sich auch in realen wirtschaftlichen Entscheidungen bemerkbar machen. Ziel ist, experimentelle Erkenntnisse auf die Wirtschaftspraxis anzuwenden und somit ein erweitertes Verständnis davon zu erlangen, wie moralische Argumente wirtschaftliches Handeln beeinflussen.

Demgegenüber liegt der Einwand nahe, dass die Soziologie seit jeher untersucht, wie moralische Argumente des sozialen Umfelds (breiter gefasst als Werte und Normen) auf menschliches Handeln einwirken. Es ist nicht Ziel dieses Artikels, diese breite soziologische Diskussion erschöpfend wiederzugeben. Wegweisend waren die Klassiker, etwa Durkheim, der Moral definierte als Rücknahme individueller Selbstinteressen zugunsten gesellschaftlicher Interessen und Normen (Durkheim 1953, S. 37, 50 f.). Weber (1978; vgl. auch Hirschman 1977) zeigte, wie stetes Gewinnstreben zum Kapitalismus führte, indem es als gottgefällig und erst damit als gesellschaftlich akzeptabel redefiniert wurde. Parsons (1937) sah Handeln als prinzipiell normativ beeinflusst, was der Soziologie den Vorwurf der „Oversocialized Conception of Man“ einbrachte (Wrong 1961). Zelizer 
(1978, 1979, 1981, 1985), Titmuss (1971) und Healy $(2004,2006)$ untersuchten in jüngerer Vergangenheit, inwiefern bestimmte „Güter“, beispielsweise Organe und Blutspenden, Kinder oder Lebensversicherungen, nicht nur nach Marktkriterien gehandelt werden, sondern dem Markt aufgrund moralischer Bedenken, die gesellschaftlich schwanken, entzogen und zugeführt werden. Stellvertretend für viele Soziologen und auf diesen Forschungsergebnissen aufbauend, forderte Etzioni (1988), menschliches Handeln aus der Hin- und Hergerissenheit zwischen ,self-interest and the pleasure principle on one hand, and powerful moral commitments on the other" (Etzioni 2003, S. 133) zu verstehen.

Doch trotz der bisherigen soziologischen Diskussion zu Moral bleibt unklar, ,how ethical questions actually arise within economic life, how economic actors respond to them, and what effects those responses have“ (Zelizer 2007, S.9). Wie moralische Überlegungen wirtschaftliche Interessenwahrnehmung beeinflussen, haben demgegenüber Verhaltensökonomen, zumindest in Experimenten, recht genau nachgezeichnet. Wären deren Ergebnisse auf reales ökonomisches Handeln übertragbar, könnte damit ein Teil der oben formulierten Fragen bezüglich der Bedeutung von Moral für wirtschaftliches Handeln beantwortet werden. Diekmann (2008, S. 529) meint jedoch, dass in der „Soziologie von diesen Entwicklungen kaum Kenntnis genommen wird. Die Soziologie wäre gut beraten, Ergebnisse der experimentellen Ökonomie aufzunehmen und in einen Dialog einzutreten“. Verhaltensökonomen dagegen haben noch nicht untersucht, inwiefern ihr experimentell beobachtbares Verhalten auf die Realität übertragbar ist.

Tatsächlich ist dem Autor dieses Artikels keine Studie bekannt, die typische wirtschaftliche Entscheidungen rekonstruiert, um empirisch zu untersuchen, wie moralische Argumente wirtschaftliches Handeln beeinflussen. Deswegen lohnt es sich, die soziologische und verhaltensökonomische Forschung zu Moral zusammenzuführen und empirisch zu untersuchen, wie moralische Argumente auf wirtschaftliches Handeln einwirken. Im Folgenden werde ich beschreiben, mit welcher Vorgehensweise dieses Forschungsprojekt dies versuchte. Daraufhin werde ich die wichtigsten Ergebnisse verhaltensökonomischer Experimente darlegen und im Anschluss zeigen, ob und inwiefern die Ergebnisse des Ultimatum-, Diktator- und Kollektivgutspiels sich in der Realität wirtschaftlichen Handelns wiederfinden.

\section{Methodologie}

Das diesem Artikel zugrunde liegende Forschungsprojekt wollte systematisch der Frage nachgehen, ob und wie moralische Argumente in wirtschaftlichen Zusammenhängen Unternehmer beeinflussen und untersuchte darum Diskussionen um Produktionsverlagerungen. Aus einer Liste von zweiundzwanzig Unternehmen, in denen nach Ansicht der IG Metall (Interview 04.01.2007) und des Arbeitgeberverbandes Gesamtmetall (Interview 04.04.2007) moralische Argumente die Pläne der Geschäftsleitung erkennbar beeinflussten oder nicht beeinflussten, wurden fünf besonders anschauliche Beispiele ausgewählt. Diese werden hier erörtert.

Anstatt bestimmte Hypothesen zu testen oder zu widerlegen, sollte zunächst möglichst unbefangen an die Empirie herangetreten werden (auch wenn dies nie restlos möglich ist), um daraus auf theoretisch relevante Sachverhalte zu schließen (vgl. Glaser und Strauss 
1967; Strauss und Corbin 1990). Ziel des Forschungsprojektes war, eine realistische Konzeption der Beeinflussung wirtschaftlichen Handelns durch „heuristische Fallstudien“ möglichst induktiv aus der Empirie zu entwickeln (vgl. Eckstein 1975, S. 104 ff.).

Es schien geeignet, Diskussionen um Produktionsverlagerung zu analysieren, denn Verlagerungen treffen die Belegschaft des Heimatstandortes in der Regel besonders hart. Die deswegen vorgebrachten moralischen Argumente, dass der Verlagernde die Interessen der Heimatbelegschaft berücksichtigen solle, lassen sich besser recherchieren als Argumente in rein unternehmensinternen und informellen Verhandlungen. Denn Produktionsverlagerungsdiskussionen werden oft in der regionalen Öffentlichkeit ausgetragen und die damals gemachten Argumente sind durch Flugblätter, Zeitungsartikel, Pressemitteilungen, Briefe etc. größtenteils archiviert. Anhand dieser Dokumente rekonstruierte eine Prozessanalyse, wer an welchem Punkt welche Argumente vorbrachte und wie sich der Verhandlungsverlauf daraufhin entwickelte (vgl. zur Prozessanalyse George und Bennett 2005; Hall 2007). Anschließend sollten Interviews mit allen Beteiligten Fragen klären, die eine Dokumentenanalyse nicht abdeckte (vgl. zur Interviewführung Gläser und Laudel 2004). Unterschiedliche Aussagen wurden mit den damaligen Dokumenten und mit weiteren Interviews abgeglichen, da die Interpretation des Forschers ansonsten vollständig daran leidet, das „Konstrukt eines Konstrukts“ zu sein (vgl. Aspers 2004; Schütz 1932, 1971, S. 5 ff.). Allen Beteiligten wurde völlige Anonymität zugesichert, ihre Aussagen sind darum nur anonym zitiert. Alle Namen sind Pseudonyme; Zeitungsartikel, aus denen Informationen stammen, können nicht mit der Quelle zitiert werden. Da es sich um vertrauliche Informationen handelt, musste im Zweifel strikte Anonymisierung Vorrang haben. Einzelne Aspekte können auf Verlangen eingesehen werden, denn die genutzten Quellen und Interviews sind schriftlich und als Tondokumente archiviert.

Dieses Vorgehen hat Schwächen. Erstens kann man niemals restlos feststellen, was die wirklichen Motive hinter Handlungen sind. Dies muss mindestens daran scheitern, dass Akteure die „letztlichen“ Gründe ihres Handelns selbst nicht kennen (Freud 1992; Wittgenstein 1953). Ziel war darum auch nicht zu psychologisieren und zu verstehen, was ,wirklich“ in Menschen vorgeht, sondern inwiefern Akteure in ihrem sichtbaren Verhalten ihre Interessen ein Stück weit zurücknehmen, um gesellschaftliche Interessen und Normen zu beachten - und sich in diesem Sinne gemäß Durkheims Definition moralisch verhalten. Das zweite Problem eines solchen qualitativen Forschungsdesigns ist seine mangelnde Generalisierbarkeit. Fallstudien können nie zeigen, was typisch ist, sondern immer nur, was möglich ist (vgl. Lijphard 1975; Gerring 2004; George und Bennett 2005). Darum kann diese Untersuchung nicht die statistische Wahrscheinlichkeit angeben, mit der die zutage geförderten moralischen Einflüsse auch in anderen Unternehmen wirken oder generell Produktionsverlagerungen beeinflussen.

Gerade aufgrund der mangelnden Verallgemeinerbarkeit von Fallstudien kommt jedoch die Komplementarität zu verhaltensökonomischen Experimenten ins Spiel, denn diese zeigen wegen des kontrollierten und reproduzierbaren Laborsettings gerade was typisch ist. So ähneln sich die grundlegenden Ergebnisse verhaltensökonomischer Experimente nicht nur über verschiedene moderne Gesellschaften, sondern sogar über verschiedene Stammesgesellschaften hinweg (Henrich et al. 2001; Herrmann et al. 2008). Nicht nur inhaltlich, wie die Einleitung knapp zu umreißen versuchte, sondern auch methodologisch bietet es sich somit an, Forschung zu der Frage zu kombinieren, wie moralische 
Argumente Handeln beeinflussen. Ich stelle im Folgenden vor, was die bisherige verhaltensökonomische Forschung zu dieser Frage beigetragen hat und vergleiche diese Befunde mit realen Geschehnissen in Diskussionen um Produktionsverlagerung.

\section{Verhaltensökonomische Experimente, soziologische Erkenntnisse}

Neoklassische Ökonomen setzen eigeninteressierte Nutzenmaximierer als Grundbausteine ihrer Handlungstheorie voraus, was Verhaltensökonomen aufgrund der Ergebnisse ihrer Experimente kritisieren, denn: ,in this paradigm, ethics plays no role in motivating human behavior“ (Gintis et al. 2005, S. 3 f.). Gintis (2010, S. 35) fordert darum: „the human predisposition to internalize social norms, and more generally to include ethical values as arguments in personal preference orderings, should be included in analytical models of strategic interaction." Doch für ein solchermaßen erweitertes Modell menschlichen Handelns müsste bekannt sein, wann Moral und die entsprechenden Argumente Handeln beeinflussen. Unter welchen Umständen dies geschieht, zeigen im Folgenden drei klassische Experimente von Verhaltensökonomen. Darin „spielen“ Menschen mit anderen, die sie nicht sehen und auch nicht persönlich kennenlernen. Anonymität stellen diese Experimente sicher, indem die Teilnehmer lediglich über Computermonitore interagieren. So können persönliche Sympathien oder ähnliche Verzerrungen nicht die Ergebnisse beeinflussen.

\subsection{Ultimatumspiel}

Das bekannteste Experiment, um die Bedeutung von Moral für menschliches Handeln darzustellen, ist das sogenannte Ultimatum-Spiel. Darin bekommt einer von zwei anonymen Spielern eine Geldmenge, beispielsweise $100 €$ in Fünfeuroscheinen. Dieser „Verteiler" kann einem Mitspieler (dem Empfänger) eine beliebige Menge davon abgeben und den Rest für sich behalten, aber nur unter der Bedingung, dass der Empfänger dies akzeptiert. Denn dem Empfänger steht es frei, den Austausch abzulehnen, wenn die angebotene Geldmenge seiner Meinung nach zu gering ist, womit beide leer ausgehen. Wirtschaftlich rational wäre es für den Empfänger, jede noch so kleine Geldsumme anzunehmen, da er damit immer noch mehr bekommt, als wenn er den Austausch ablehnt. Da der Verteiler dies vorhersehen sollte, müsste er entsprechend eine möglichst geringe Summe anbieten (beispielsweise einen Fünfeuroschein), die dann vom Empfänger angenommen wird. Diese Lösung wäre eine sowohl teilspielperfekte als auch pareto-optimale Nash-Gleichgewichtsstrategie (vgl. Diekmann 2008, S. 538).

Doch in der Realität verhalten sich Spieler selten so. Vielmehr bieten Verteiler typischerweise 40-50\% einer beliebigen Summe an. Und auch wenn sie das nach einem strikten Eigennutzpostulat weder sollten noch müssten, tun sie in der Realität gut daran. Denn Empfänger lassen den Austausch oft platzen, wenn Verteiler weniger als ca. 30-40\% der Gesamtsumme anbieten. Dies ist wiederum für ein striktes Eigennutzpostulat nicht erklärbar, denn offenbar verzichten Menschen oft auf circa $30 \%$ einer beliebigen Summe, um Verhalten zu bestrafen, welches sie als unmoralisch wahrnehmen, auch wenn sie die Person, die sie bestrafen, nicht kennen und nie treffen werden. Natürlich variieren die 
Ergebnisse, je nach genauem Spielaufbau und kulturellem Hintergrund der Spielenden. Doch die grundlegenden Resultate replizierten Forscher in mehreren Ländern mit bis zu zwei Monatseinkommen. Danach lehnen Spieler $20 \%$ einer angebotenen Summe mit 50prozentiger Wahrscheinlichkeit ab und bieten in der Regel 40-50\% an (Camerer 1997, S. 169; Gintis et al. 2005, S. 11 ff.; vgl. klassisch Güth et al. 1982; Kahneman et al. 1986; für eine gute und kurze Einführung, vgl. Thaler 1992, S. 21 ff.).

\subsection{Diktatorspiel}

Das typische Verhalten beim Ultimatumspiel erlaubt verschiedene Interpretationen menschlicher Motivation. Möglicherweise teilt der Verteiler lediglich nur deshalb, weil er aus richtig verstandenem Eigennutz fürchtet, ansonsten leer auszugehen. Er könnte schließlich voraussehen, dass der Mitspieler den Austausch aus Fairnessüberlegungen, wenn auch materiell irrational, ablehnt. Dass dies nicht die ganze Erklärung ist, zeigt das Diktatorspiel, der zweite Klassiker verhaltensökonomischer Verteilungsspiele. Darin muss der Empfänger den vorgeschlagenen Austausch annehmen, selbst wenn ihm das Verteilungsergebnis nicht passt. Diesmal hat der Verteiler somit keinen Anreiz, etwas abzugeben, außer den Anreiz, einer entsprechenden moralischen Norm zu folgen. Dies macht sich durch geringere Abgaben bemerkbar. In einem klassischen experimentellen Setup gaben im Diktatorspiel nur noch 22\% der Mitspieler die Hälfte der Summe oder mehr ab, während noch $65 \%$ dies im Ultimatumspiel taten. 36\% der Mitspieler behielten alles für sich, während dies im Ultimatumspiel niemand machte. Wurde dieses Experiment mit 10, statt mit 5 \$ wiederholt, gaben im Diktatorspiel nur noch $21 \%$ der Spieler die Hälfte ab (und niemand mehr als die Hälfte), während im Ultimatumspiel mit 10 \$ noch ganze $75 \%$ mindestens die Hälfte abgaben; $21 \%$ der Mitspieler gaben im Diktatorspiel mit 10 \$ sogar nichts $\mathrm{ab}$, während im entsprechenden Ultimatumspiel niemand alles für sich behielt (Forsythe et al. 1994, S.362 f.).

Es ist also nicht nur Orientierung an moralischen Normen, die Menschen im Ultimatumspiel beeinflusst, denn dann würden sie genau so viel abgeben wie im Diktatorspiel. Es ist aber auch Orientierung an moralischen Normen, denn zwar geben Spieler im Diktatorspiel im Unterschied zum Ultimatumspiel nicht mehr ca. 40-50\% der Gesamtsumme ab, doch immer noch geben sie im Schnitt 20-30\% ab, was materiell gesehen irrational ist (Camerer 1997: 169; Kahneman et al. 1986; Thaler 1992, S. 25). Zwar gibt es im Diktatorspiel auch einige Menschen, die gar nichts abgeben; auf der anderen Seite „verschenken“ viele Mitspieler jedoch sogar die Hälfte ihrer Summe. Dies gilt, obschon Verteiler und Empfänger anonym sind und auch wenn klar ist, dass sie nicht mehr interagieren werden (sogenannte „One-shot“-Spiele).

Altruistisch abzugeben scheint nicht nur anerzogen zu sein, denn ,,auch Kinder verhalten sich im Diktatorspiel altruistisch, wobei die Abgabequote mit dem Alter und dem Sozialstatus der Eltern ansteigt“" (Diekmann 2008, S. 532). Zwar zeigten Versuche in verschiedenen Gesellschaften, dass die Ergebnisse variieren, doch in keiner Gesellschaft, die im umfangreichen Sammelband von Henrich et al. (2004, S. 5) analysiert wurde, fanden die Forscher restlos selbstinteressiertes Verhalten. Interessanterweise teilen Menschen umso mehr, je mehr sie in ihren Gesellschaften kooperieren und umso stärker ihre Gesell- 
schaften Güter über den Markt verteilen, wie Versuche in siebzehn Stammesgesellschaften zeigen (Henrich et al. 2001, S. 76).

\subsection{Kollektivgüterspiele}

Public-Goods-Spiele (Kollektivgüterspiele) testen als dritter Klassiker verhaltensökonomischer Forschung, wie Moral als zumindest kurzfristiges Zurückstellen selbstbezogener materieller Interessen menschliches Handeln beeinflusst. In diesen Spielen können Mitspieler ein optimales Resultat erreichen, wenn sie zusammenarbeiten. Jeder Mitspieler hat jedoch individuell einen Anreiz, sich egoistisch zu verhalten. Beispielsweise bekommt jeder von vier anonymen Mitspielern eine Summe von $20 €$. Diese kann er für sich behalten oder einen beliebigen Anteil davon in einen gemeinsamen „Topf“ investieren, der ein Kollektivgut repräsentiert. Alles, was investiert wird, wird beispielsweise mit 1,4 multipliziert und dann gleichmäßig unter allen Mitspielern aufgeteilt. Jeder Einzelne bekommt somit bei einer Multiplikation von 1,4 und 4 Mitspielern von seiner Investition nur $35 \%$ zurück, dafür bekommen alle anderen Spieler ebenfalls $35 \%$ jeder individuellen Investition. Eine volle Investition jedes einzelnen Mitspielers ist somit pareto-optimal, alle profitieren und keiner ist schlechter gestellt. Für jeden Einzelnen bedeutet seine individuelle Investition jedoch einen Verlust. Das Nash-Gleichgewicht in dieser Strategie besteht somit darin, dass niemand investiert, denn individuell und materiell rational ist es für jeden Mitspieler, nichts in den Topf einzugeben und zu hoffen, dass seine Mitspieler möglichst viel beisteuern, um von der mit 1,4 multiplizierten und an alle ausgeschütteten Summe zu profitieren.

Doch erneut verhalten sich die meisten Spieler nicht in diesem Sinne individuell nutzenmaximierend. Meist investieren sie stattdessen 40-60\% ihrer Summe in den gemeinsamen Topf. Wie die anderen Spiele auch, kann das Kollektivgutspiel ebenfalls in mehreren Runden gespielt werden. Dabei wird nach jeder Runde anonym erkenntlich, wie viel die anderen Mitspieler in den Topf investiert haben. Mit zunehmender Rundenzahl sinkt in der Regel das Investment. Weitere Experimente zeigten, dass dies geschieht, da die Investierenden sich zunehmend zurückhalten, um diejenigen zu bestrafen, die nicht investieren (vgl. Gintis et al. 2005, S. 15 ff.; Diekmann 2008, S. 532; Thaler 1992, S. 10 f.; vgl. für ein Zwei-Personen-Spiel, das einem Arbeitsvertrag nachempfunden ist Fehr et al. 1993).

Wenn die Investierenden dagegen die Nicht-Investierenden nicht nur durch Zurückhaltung bestrafen können, bleibt das Einzahlungsverhalten konstant hoch. In einem Spiel, das Einzahlungen mit 1,6 multiplizierte, konnten Mitspieler einen ihrer Punkte opfern, um einem nicht kooperierenden Mitspieler drei Punkte abzuziehen. Dies nutzten in zehn Runden $84 \%$ der Mitspieler mindestens einmal, wobei sie umso stärker sanktionierten, umso weniger ein Mitspieler kooperierte. 94\% mehr Mitspieler investierten bei dieser Variante gegenüber einer ohne Bestrafungsmöglichkeit. Wichtig ist, dass auch hier die Bestrafung materiell gesehen irrational ist, da es für den Einzelnen mehr kostet zu bestrafen als er an materiellem Nutzen dadurch gewinnt, wobei von dem Nutzen der Bestrafung dafür die ganze Gruppe profitiert. Trotzdem bestrafen Spieler selbst dann noch, wenn sie mit wechselnden Mitspielern spielen, die sie somit nicht „erziehen“ können (Fehr und Gächter 2002, S. 137 f.). Sie bestrafen somit nicht, weil sie die Anderen zwingen wollen zu kooperieren, um letztlich selbst zu profitieren. Ein im Sinne der klassischen 
ökonomischen Theorie spielender Mitspieler sollte in jedem Fall hoffen, dass seine Mitspieler bestrafen (aber nicht ihn) und er von der dadurch gestiegenen Investition profitiert. Jedoch sollte jeder Spieler selbst nicht bestrafen, da ihn dies individuell immer mehr kostet als nutzt.

Doch auch bei diesem Experiment verhalten sich Menschen nicht in diesem Sinne rational. Forscher führten es in verschiedenen Kulturkreisen durch und immer wieder wurden Mitspieler, die nicht kooperierten, altruistisch sanktioniert. Menschen handeln somit nicht per se oder ,natürlicherweise“ egoistisch, sondern erst, wenn andere dies auch tun und sie diese „Ungerechtigkeit“ nicht anders als durch Nicht-Investition bestrafen können. Egoismus breitet sich dann von einer kleinen Anfangsgruppe auf die gesamte Gruppe aus. Wenn man es jedoch ermöglicht, Nicht-Kooperation zu bestrafen, dann finden sich auch Individuen, die dies altruistisch tun. Dadurch haben nicht nur diejenigen, die bestrafen können, nicht mehr das Gefühl, dass sie „die Dummen“ sind, da sie kooperieren. Auch haben Egoisten einen Anreiz zu kooperieren, wenn auch nur, um nicht bestraft zu werden. Interessanterweise zeigt sich dann sogar, dass es in späteren Spielrunden gar nicht mehr nötig ist, kostenträchtig zu bestrafen, da schon die Möglichkeit der Bestrafung kooperatives Verhalten bringt (vgl. Fehr und Fischbacher 2005, S. 169 ff.).

\subsection{Was die Soziologie zu verhaltensökonomischen Experimenten beitragen muss}

Elinor Ostrom (2005, S. 254; vgl. auch Gintis et al. 2005, S. 15) faßt diese Ergebnisse zusammen: „Laboratory experiments of social dilemmas, trust games, dictator games, and ultimatum games repeatedly find higher-than-predicted cooperative behavior that cannot be explained by theories assuming the existence of only rational egoists." Die meisten Spieler verhalten sich stattdessen reziprozitär, sie kooperieren, insofern ihre Mitspieler kooperieren. Dabei gibt es wiederum reziprozitäre Spieler, die ihre Kooperation sofort einstellen, sobald jemand nicht kooperiert, und andere, die auch dann noch kooperieren, wenn andere dies über mehrere Runden nicht mehr tun, während die meisten Spieler dazwischenfallen (Kahan 2005, S. 345). Doch Verhaltensökonomen betrachten es als fraglich, ob, wie und inwiefern dieses von ihnen experimentell aufgedeckte Verhalten auf tatsächliches wirtschaftliches Handeln verallgemeinerbar ist oder ob es ein Artefakt der Laborsituation ist (vgl. Gintis et al. 2005, S. 5). Während Verhaltensökonomen versuchen, die ,contaminating conditions of the real world“ aus dem Labor herauszuhalten, werden sie von Soziologen genau dafür kritisiert:

Behavioural economics stripped human beings naked of their social relations and connections, took away their language and with it their scientifically uninformed views about themselves and put them in a vacuum, as it were, where lead balls and feathers take exactly the same time to fall, revealing and confirming natural laws otherwise hidden by the contaminating conditions of the real world. (Streeck 2010, S. 391)

Weil insofern unklar ist, ob Orientierung an Moral ein Artefakt der Laborsituation ist, fordern auch Verhaltensökonomen soziologische Feldstudien, die das experimentell zutage geförderte Verhalten mit real stattgefundenem ökonomischem Handeln in seinem realen 
sozialen Umfeld abgleichen (Henrich et al. 2001, S.77). Greiner und Ockenfels (2009, S. 238) bemerken beispielsweise:

Theorie und Laborexperiment allein [reichen] nicht aus, um der Komplexität realer sozialer Phänomene in bestimmten Kontexten umfassend gerecht zu werden. Der Grund ist, dass Modelle stets von der Realität und oft auch von relevanten Kontexten abstrahieren, um Kausalitäten aufdecken zu können. Daher ist komplementär zur Mikrofundierung in der Ökonomik der Einsatz von Fallstudien und anderer empirischer und theoretischer Methoden notwendig, um die relevanten Komplexitäten adressieren zu können.

Auch Diekmann (2008, S.530 f.) merkt an: „Skepsis gegenüber der Künstlichkeit von Laborexperimenten kann durch Feldexperimente im ,natürlichen' sozialen Umfeld ... begegnet werden." Doch weder Soziologen (siehe Einleitung) noch Ökonomen haben bisher entsprechende Studien durchgeführt, um zur Lösung der angesprochenen Probleme beizutragen. Elinor Ostrom (1990) untersucht zwar anhand von Fallstudien, inwiefern Gemeinschaften Kollektivgutprobleme lösen. Sie untersucht aber nicht, wie im Sinne der oben präsentierten Spiele in modernen, kapitalistischen Gesellschaften Unternehmerentscheidungen moralisch beeinflusst werden. Genau dies untersuchte darum das oben beschriebene Forschungsprojekt. Dieses war zu Laboruntersuchungen komplementär, denn die Situation in der Realität ist immer vielschichtiger als in den Versuchsbedingungen eines Experiments, was einerseits die Schwäche qualitativer Analysen ist und überhaupt erst Experimente notwendig macht. Auf der anderen Seite kann nur Feldbeobachtung zeigen, inwiefern die „im Prinzip“ vorhandene menschliche Neigung, zumindest partiell seine eigenen Interessen zurückzunehmen, auch real stattgefundenes wirtschaftliches Handeln beeinflusst. Inwieweit dies in der Praxis geschieht, zeigen Diskussionen um Produktionsverlagerungen. Ähnlich wie in verhaltensökonomischen Experimenten und trotz aller Unterschiede gegenüber diesen, besteht auch in Produktionsverlagerungsdiskussionen die Möglichkeit von Seiten der Unternehmensleitung, wirtschaftliche Interessen ein Stück weit und zumindest kurzfristig zurückzunehmen, um moralischen Ansprüchen des sozialen Umfelds entgegenzukommen. In welchem Maße und wie dies geschieht, zeigen folgende Unternehmensstudien.

\section{Vom Labor ins Feld: Diskussionen um Produktionsverlagerungen}

\subsection{Diktatorspiel-Situationen: Das Unternehmen Müller}

Moralischem Verhalten im Diktatorspiel entsprechen Situationen, in denen Menschen ihren Nutzen insofern nicht maximieren, als sie sich mit einem bestimmten materiellen Gewinn zufriedengeben und anderen etwas abgeben, obwohl sie dazu nicht verpflichtet sind. Noch weniger als in einem anonymen Experiment ist in der sozialen Realität zwar restlos belegbar, dass jemand tatsächlich etwas abgibt, da er sich an moralischen Argumenten orientiert. Schließlich ist es auch immer möglich, dass jemand durch (schein)moralisches Verhalten hofft, etwas zurückzubekommen, und sei es Anerkennung. Doch innerhalb dieser Gegensätze ist das Unternehmen Müller ein interessanter Fall. Der alt- 
eingesessene Patriarch Peter Müller leitete dieses Familienunternehmen und war nach Meinung der Arbeitnehmervertreter eng an seine Belegschaft gebunden, mit der er seit mehreren Generationen zusammenarbeitete.

Doch da sich die Rendite (EBIT) ${ }^{1}$ seines Unternehmens im Laufe der Zeit auf circa $1,9 \%$ verringert hatte, forderte er von seinen Mitarbeitern Konzessionen, um nicht zu verlagern. Während der entsprechenden Verlagerungs- und Konzessionsdiskussion definierte er in einem Brief, wie viel Gewinn er anstrebe und setzte sich damit öffentlich eine Gewinnobergrenze, ab der er bereit sei, von weiteren Konzessionsforderungen abzusehen: „Angemessen ist ein Gewinn dann, wenn er 6\% vom Umsatz übersteigt.“ Ab einem EBIT von $4 \%$ beteiligte er darum seine Belegschaft am Unternehmensgewinn. Umgekehrt äußerte er, ,,wenn die Umsatzrendite unter $4 \%$ sinkt, dann können wir auch keinen Bonus bezahlen“ (Müller 1. Interview: 23. Min.). Als ich ihn fragte, warum er sich mit einem bestimmten Gewinn zufriedengebe, statt zu versuchen, diesen zu maximieren, antwortete er:

Weil ich der Meinung bin, dass man bei einem entsprechenden Ergebnis, an dem ja die Belegschaft mitgewirkt hat - nicht ausschließlich, da kommen ja viele andere Dinge zusammen - dass man die durchaus daran teilhaben lassen sollte, weil sie daran mitgewirkt hat und das ja auch dem Unternehmen, wenn es ordentliche Ergebnisse hat, auch gar nicht wehtut. (Müller 1. Interview: 36. Min.)

Insbesondere wenn man, wie ich das seit 27 Jahren macht, da fühlt man sich schon für die Mitarbeiter verantwortlich. Da sind ja auch Familien dahinter, Kinder und Sicherheiten und, also, wo finden die nen Arbeitsplatz? Also, da fühlt man sich schon verantwortlich. [...] Also, man hat sie gegenüber dem Kunden, die Verantwortung, man hat sie aber auch in der Region hier, wo die Menschen, die hier arbeiten, wohnen oder die Stadt, die die Steuern braucht. (Müller 1. Interview: 13. Min.)

Im Privatleben würde solch eine Argumentation vielleicht nicht erstaunen. Es ist aber interessant, sie auch in wirtschaftlichem Handeln zu finden; schließlich hätte Müller auch argumentieren können, das legitime Ziel eines Unternehmens sei nun einmal Gewinnmaximierung (vgl. Friedman 2001). Als ich ihn noch einmal explizit fragte, ob er nicht auch eine höhere Rendite anstreben könne, antwortete er: „Ja gut, ok, ja, da ist man vielleicht als Mittelständler, und vor allem, wenn man etwas älter ist, wie ich, da ist man etwas nachsichtiger“ (Müller 1. Interview: 32. Min.). Die zuständige Gewerkschaft bestätigte, dass diese Haltung tatsächlich typisch für Müller sei, obwohl er unnachgiebig seine wirtschaftlichen Interessen vertrete, wenn es um das Überleben seines Unternehmens ging (Müller 2. Interview).

Augenscheinlich ging es Müller nicht darum, seinen Gewinn zu maximieren, sondern er wollte ,genug“" verdienen, um seinen Lebensstandard zu sichern und notwendige Investitionen zu tätigen. An die Stelle eines Maximierungskalküls trat die Vorstellung eines

1 EBIT ist eine Kennzahl, die den Unternehmensgewinn vor Steuern, Finanzergebnis (zum Beispiel Zinsen) und außerordentlichen Aufwendungen wiedergibt. Der EBIT gibt also das Unternehmensergebnis wieder, bereinigt um Kosten und Erträge, die nicht aus der eigentlichen betrieblichen Tätigkeit entstanden sind. Er ermöglicht, den Gewinn verschiedener Unternehmen zu vergleichen, auch wenn diese unterschiedlich hohe Zins- und Steuerlast zu tragen haben. 
branchenspezifischen Normalgewinns, der zur Deckung seines Lebensbedarfs ausreichen sollte. Sowohl Arbeitnehmervertreter als auch Management führten dies darauf zurück, dass er eine moralische Verantwortung gegenüber seiner Belegschaft höher gewichtete als das Ziel, materiellen Wert zu maximieren. Dies reflektiert den Unterschied zwischen einer „kapitalistischen“"Wirtschaftsethik, nach der ein Gewinn nie hoch genug sein kann, und einer „traditionalen“ Wirtschaftsethik, nach der ein Gewinn situationsangemessen sein muss und darüber hinaus genutzt wird, um moralischen Pflichten nachzukommen, die in sozialen Bindungen erwachsen (vgl. Polanyi 1944, S.47; Weber 1988, S. 44-55). Dabei war weniger relevant, ob Müller verlagerte oder nicht. Dass er von einer Verlagerung absah, könnte auch mit hoher Risikoaversion erklärt werden. In Anbetracht verhaltensökonomischer Experimente ist vielmehr die Neigung von Interesse, anderen etwas abzugeben und sich eine Gewinnobergrenze zu setzen. Nicht nur im Diktatorspiel, sondern auch in tatsächlichem Unternehmerhandeln lässt sich somit die Motivation erkennen, anderen etwas abzugeben, solange man dabei selbst nicht ,zu kurz kommt“"

\subsection{Ultimatumspiel-Situationen: Das Unternehmen Steche}

Wie schon dargelegt, testet das Diktatorspiel, ob Menschen etwas abgeben, obwohl sie davon keinen materiellen Nutzen erwarten können. Dagegen testet das Ultimatumspiel, ob Menschen Angebote ablehnen, von denen sie zwar ökonomisch profitieren, die sie jedoch unfair finden. Dies geschah im Unternehmen Steche. Ein ausländischer Finanzinvestor kaufte es und verlangte kurz darauf Konzessionen von der Belegschaft, um nicht zu verlagern. Die Arbeitnehmervertreter willigten ein. Doch ihr Vertrauen, dass deswegen nicht verlagert würde, enttäuschte der Vorstand Hermann Thüren, indem er kurze Zeit später und trotz gegenteiliger Versprechen eine Verlagerung ankündigte, falls es nicht zu weiteren Konzessionen von der Belegschaft käme. Indem Thüren die Belegschaft dazu bewog, zu kooperieren, dann selbst aber implizite Versprechen brach, verhielt er sich nach einem neoklassisch-nutzenmaximierenden Verständnis rational. Obwohl demgegenüber Betriebsrat und Gewerkschaft betonten, dass es nun ihrerseits ökonomisch gesehen sinnvoll wäre, Thürens Forderungen nach erneuten Konzessionen zuzustimmen, denn dem Unternehmen ging es wirklich schlecht, äußerten sie:

Da ich Aufsichtsratsvorsitzender war, war ich mit denen immer im Gespräch. Ich kannte die Zahlen. Im Prinzip hätte ich schon fast sagen müssen: ,Wir müssen eigentlich nach Osteuropa gehen. 'Wie wollen wir das sonst noch hinkriegen hier? Das haben wir natürlich nicht gesagt. (Steche 1. Interview: 26-28. Min.)

Da haben wir nicht mehr mitgemacht. Wir haben einen Tarifvertrag. Wir sind Verpflichtungen eingegangen hier zu investieren, hier auch Projekte anzuschieben für neue Produktionsmethoden, Produktionslinien, Produktentwicklung zu betreiben, Qualifizierung der Beschäftigten. Es war nichts passiert. (Steche 3. Interview: 30-50.Min.)

Da sich Betriebsrat und Gewerkschaft betrogen fühlten, wollten sie nicht mehr kooperieren. Der Betriebsrat äußerte gar zu Thürens Konzessionsforderungen: „Mit mir nicht, kein Stück!“ und fügte trotzig hinzu: „Dann macht mal schön die Insolvenz“ (Steche 2. Interview: 0:45:16 und 0:48:23). Im Ultimatumspiel lehnen Empfänger Angebote ab, die 
sie für unangemessen niedrig halten, obwohl sie materiell immer noch davon profitieren würden. Damit bestrafen sie zu ihrem eigenen materiellen Schaden sich selbst und den Anbieter, da dieser in ihren Augen moralische Verhaltensregeln missachtet. Demselben Prinzip folgte die Ablehnung von Steches Arbeitnehmervertretern, welche das als unmoralisch angesehene Verhalten Thürens bestrafen wollten, selbst wenn dies für alle Beteiligten wirtschaftlich desaströse Folgen hatte. In diesem Sinne lehnen Menschen nicht nur im Experiment Angebote ab, die sie zwar ökonomisch besserstellen, jedoch ihr Gerechtigkeitsempfinden verletzen, sondern verhalten sich so auch in der Praxis.

\subsection{Kollektivgüterspiel-Situationen}

\subsubsection{Tehnwolder - Soziale Vorbedingungen erfolgreicher Kooperation}

Eine reale Situation entspricht einem kooperativen Kollektivgüterspiel, wenn Arbeitnehmer und Arbeitgeber in einen gemeinsamen Pool investieren, was ein besseres Ergebnis ermöglicht, obwohl jede der beiden Seiten auf den Einzahlungen der anderen Seite Trittbrett fahren könnte. Solch eine Situation fand sich im Unternehmen Tehnwolder, welches der alteingesessene Patriarch Reinhard Tehnwolder leitete. Im Unterschied zu Müller konnte dieser jedoch nicht auf einen hohen Gewinn verzichten, auch wenn er persönlich vielleicht dazu bereit gewesen wäre. Denn ein amerikanischer Investor hielt zwei Drittel der Firmenanteile Tehnwolders und ließ die operative Unternehmensführung nur unter der Bedingung hohen Profits in den Händen des Oberhaupts der Familie Tehnwolder. Der Betriebsrat bemerkte über Reinhard Tehnwolder, er habe eine:

Verbundenheit zum Unternehmen, die unbestritten ist. Und am Heimatstandort gibt es immer welche, die zu seiner Frau oder ihm ankommen und sagen: ,Hilf uns, weil im Geschäft ist der böse oder der Meister...'Also auch auf sehr kleiner Ebene, dass das dann zu ihm hoch getragen wird. Ich glaube, man kann es sich gar nicht vorstellen, wenn man das Kleine, das Ländliche gar nicht kennt. Dann tut man sich schon schwer, so etwas zu glauben. So funktionieren doch Unternehmen nicht! (Tehnwolder 1. Interview: 01:23:57)

Reinhard Tehnwolders soziale Einbettung führte jedoch nicht zu sentimentaler und unökonomischer Irrationalität. Stattdessen erlaubte sie im Sinne eines Kollektivgutspiels zu kooperieren, was ansonsten daran scheitern würde, dass die Belegschaft ihm nicht vertrauen könnte. Der Betriebsrat beschrieb entsprechend, warum der amerikanische Investor die Unternehmensleitung bewusst bei Reinhard Tehnwolder beließ, anstatt eigene Führungskräfte in das Unternehmen zu bringen:

Wenn es dann der Finanzvorstand wäre, das wäre der Super-Gau für uns. Dann ist das Geflecht weg und dann ist auch das Vertrauen weg. Momentan haben wir das Vertrauen definitiv. Es ist ein gegenseitiges Vertrauensverhältnis. Die TehnwolderFamilie weiß, dass es für einen Mitarbeiter sehr viel bedeutet, hier zu arbeiten. Es ist ja ein langjähriges Familienunternehmen. Aber genauso wichtig ist es für die Belegschaft, dass im Vorstand jemand Tehnwolder heißt. (Tehnwolder 2. Interview: 61-62.Min.) 
Der Investor wollte somit, dass die Belegschaft ihrer Geschäftsleitung vertrauen konnte, da er dies als wirtschaftlichen Vorteil ansah, der beide Seiten motivierte, sich loyal zu verhalten und mehr als „Dienst nach Vorschrift“ zu leisten oder die andere Seite gar zu hintergehen. In diesem Sinne investierten beide Seiten in einen gemeinsamen Pool, auch wenn über Vertrauen hinaus kein Beleg dafür bestand, dass diese Investition erwidert würde. Da Belegschaft und Geschäftsleitung aber schon lange auf diese Art kooperierten, es war ein fast 100 Jahre altes Familienunternehmen, war das Arrangement stabil. Doch solch eine Kooperation nach Art eines Kollektivgüterspiels kann auch zusammenbrechen; die Bedingungen eines solchen Zusammenbruchs, wie er ebenfalls im Experiment $\mathrm{zu}$ beobachten ist, zeigen folgende zwei reale Beispiele.

\subsubsection{Wolder - Wie Kooperation zusammenbricht}

Wenn dieser Artikel bisher nur Beispiele aufführte, in denen Kooperation möglich war, auch wenn sie bei neoklassischen Verhaltensannahmen nicht zu erwarten wäre, so geschah dies, um darauf hinzuweisen, dass Menschen nicht nur im Experiment, sondern auch in real stattfindendem wirtschaftlichem Handeln kooperieren. Doch zu den entsprechenden Experimenten gehört auch, dass Menschen nicht immer auf moralische Verhaltensregeln eingehen und darum kooperieren. Im Falle des Diktator- und Ultimatumspiels wäre es banal, dies zu zeigen, denn natürlich geben Menschen nicht immer selbstlos ab und natürlich lehnen Menschen nicht immer aus Fairnessüberlegungen Angebote ab, die sie ökonomisch besserstellen. Interessant ist dagegen, warum in realen Kollektivgutsituationen Kooperation zusammenbricht. Um dies zu zeigen, illustrieren im Folgenden die Beispiele der Unternehmen Wolder und Fernlich, auf welchen sozialen Vorbedingungen Kooperation beruht. Etwas, das im Labor insofern kaum getestet werden kann, als dort soziale Randbedingungen isoliert werden.

Ähnlich wie bei Tehnwolder bestand auch in den Unternehmen Wolder und Fernlich anfangs ein Vertrauensverhältnis, das die Arbeitnehmer bewog, in einen gemeinsamen Kooperationspool einzuzahlen, indem sie sich für ihr Unternehmen mehr als nötig und mehr als nachprüfbar engagierten. Dieses Vertrauen und auch die damit einhergehende Kooperation ging jedoch verloren, als die sozialen Vorbedingungen dafür zerstört wurden. Im Folgenden werde ich herausarbeiten, unter welchen Umständen Defektion im realen Wirtschaftsleben vorkommt, da Akteure nicht unter allen Umständen darauf verzichten, ihr kurzfristiges Eigeninteresse zu verfolgen.

Ähnlich wie bei Tehnwolder vertrauten auch Wolders Arbeiter auf das Wohlwollen ihrer Geschäftsleitung. Der zuständige Gewerkschaftssekretär erinnert sich, wie Arbeiter ihm früher oft sagten: „Warum soll ich in die Gewerkschaft gehen? Ich bin doch bei Wolder" (Wolder 1. Interview: 1:15:37). Die Arbeiter vertrauten darauf, dass ihr Vorstand kooperierte, da er in der Vergangenheit erkennbar und freiwillig kurzfristige Unternehmensinteressen zurücknahm, um ihnen entgegenzukommen, und sich damit (aus ihrer Perspektive) moralisch verhielt. Beispielsweise sah der Vorstand davon ab, ein ostdeutsches Werk zu schließen, das zwar nicht so profitabel wie die anderen Werke war, dafür aber in einer strukturschwachen Region lag, in der die Arbeitnehmer kaum neue Arbeitsplätze finden würden. Zwei Betriebsräte erinnern sich, warum der damalige Vorstandsvorsitzende den Standort nicht schließen wollte: 
Da glaube ich schon, dass es soziale Aspekte waren, die da mitspielten. Da glaube ich schon, dass es die Personen, sprich der damalige Vorstandschef waren, die sagten: ,Die haben wir nach der Wende quasi zum Nulltarif bekommen, die wollen wir jetzt nicht in den Arsch treten.' Das hat nie einer in der Form ausgesprochen, aber ich denke mal, dass das die Entscheidung war. (Wolder 1. Interview: 0:53:32)

Der damalige Vorstandschef hat nichts dazu gesagt. Ich weiß aber, dass er sich im Nachhinein für uns eingesetzt hat, obwohl er uns das nie gesagt hat, da ist er nicht der Kerl dafür. Wenn er so etwas gemacht hat, dann hat er nicht damit geprahlt. (Wolder 2. Interview: 0:14:30)

Im Gegenzug arbeitete die Belegschaft nach Meinung der Geschäftsleitung besonders motiviert und leistete mehr als „Dienst nach Vorschrift“. Doch die Kooperation zwischen Arbeitnehmern und Geschäftsleitung brach zusammen, als Wolder an einen Finanzinvestor verkauft wurde: Der neue Vorstand James Layden, ein Engländer, war sozial nicht an die Heimatregion des Unternehmens gebunden und ließ auch nicht erkennen, dass er im Sinne gegenseitiger Rücksichtnahme mit der Belegschaft „kooperieren“ würde. In der Tat kündigte Layden kurz nach seinem Amtsantritt an, trotz hoher Rendite Arbeitsplätze abzubauen. Die Belegschaft sah dies als Ende der Kooperationswilligkeit der Geschäftsleitung an. Sie meinte, Layden kündige einen ,impliziten Vertrag““ auf, wonach die Belegschaft mehr als Dienst nach Vorschrift leistete und dafür ihre Arbeitsplätze gesichert seien, obwohl keines von beidem vertraglich zugesichert war und darum „nur“ auf Vertrauen beruhte. Zwei Arbeitnehmervertreter schildern, wie sie Laydens Verhalten im Gegensatz zum vorherigen Vorstand wahrnahmen:

Wir haben [früher] bei Arbeitsplatzabbau immer nach außen geguckt und haben gesagt: ,So etwas kann uns nicht passieren, waren da auch ziemlich arrogant teilweise.' Und für viele Leute brach [durch die Ankündigung des Arbeitsplatzabbaus] eine Welt zusammen. Die Eltern haben hier gearbeitet, die Kinder sind schon wieder drin, zum Teil haben die Großeltern bei Wolder gearbeitet. ,So etwas gibt es nur woanders und nicht bei Wolder', haben wir gedacht. Und auf einmal hat es uns getroffen, aus heiterem Himmel. (Wolder 1. Interview: 0:29:53)

Das war eine ganz üble Geschichte. Und da haben die gezeigt, es geht darum, Profit zu machen und nicht um die Menschen. Da haben die moralische Appelle nicht interessiert. Die haben ganz offen gesagt: ,Moral hat im Geschäft nichts zu suchen. ' Die haben einen Auftrag, bei ihnen ist Geld angelegt, die haben den Auftrag, das Geld anzulegen, die Rendite so hoch wie möglich zu erwirtschaften. Ist zwar bedauerlich, wenn Menschen auf der Strecke bleiben .... (Wolder/Tehnwolder 1. Interview: 19-20.Min.)

Die Arbeitnehmer waren erbost über die Sparvorschläge. Sie verstanden nicht, warum Layden Arbeitsplätze abbauen wollte, während Wolder fast 20\% Rendite und volle Auftragsbücher hatte. Die Belegschaft gab ihre kooperative Haltung auf und organisierte sich gewerkschaftlich, denn der Konflikt führte ihr die Notwendigkeit einer organisierten Interessenvertretung vor Augen (Wolder 1. Interview: 1:15:37). Die Belegschaft war sogar so aufgebracht, dass es zu Mordaufrufen und einer Bombendrohung gegen Layden kam. Damit einher ging, dass die Belegschaft nicht mehr intrinsisch motiviert war. Layden 
versuchte, dem mit Videobotschaften entgegenzusteuern, in denen er auf die hohe Unternehmensrendite hinwies und mitteilte, wie wichtig die Belegschaft ihm sei. Doch da sein Handeln seinen Worten widersprach, glaubte die Belegschaft ihm nicht:

Wie kommt es bei einer Montagedame an, oder bei einem Gießer oder einem Schleifer? Die sagen: ,Ist der bekloppt? Erst macht er eine Milliarde Umsatz, und was dann das Ergebnis daraus ist, das interessiert ihn doch gar nicht mehr.' Da sind die viel zu weit weg. Das ist auch gar nicht böse gemeint. Da können die gar nicht näher dran sein. Dann sehen die die Milliarde [Umsatz] und fragen sich: ,Und wofür hat er dann 1000 Leute rausgeschmissen?‘. (Wolder 1. Interview: 0:30:22 und 0:50:18) Ich behaupte, dass die Belegschaft in hohem Maße verletzt ist. Dass sie mit dem Unternehmen nichts mehr zu tun hat, außer dass sie zur Arbeit geht. (Wolder/Tehnwolder 2. Interview: 27. Min.)

Insofern ist es nicht selbstverständlich, dass Menschen in wirtschaftlichen Situationen im Sinne eines Kollektivgutspiels kooperieren. Vielmehr beruht Kooperation auf sozialen Bindungen, die in dem beschriebenen Fall mit dem Abgang eines sozial eingebundenen Vorstands nicht mehr gegeben waren. Dies widerspricht der neoklassischen Theorie, in der es diesen Unterschied gar nicht geben dürfte. Danach wären Arbeitnehmer in jedem Fall ,,interest-seekers with guile“, die auch beim ersten Vorstand nicht hätten kooperieren dürfen (vgl. Williamson 1985, S. 47 ff., 64 ff.). Analog zu den Laborexperimenten von Verhaltensökonomen zeigt sich jedoch auch in der Realität, dass Kooperation aufrechterhalten bleibt, solange im Sinne von Reziprozität beide Seiten davon ausgehen, dass die andere sie unterstützt.

\subsubsection{Fernlich - Wie Kooperation zusammenbricht}

$\mathrm{Zu}$ einem ähnlichen Zusammenbruch von Kooperation kam es im Unternehmen Fernlich. Als dieses in den 1990er Jahren einen feindlichen Übernahmeversuch abwehrte, demonstrierte seine Belegschaft noch für die Selbstständigkeit „,ihres“ Unternehmens (Fernlich 4. Interview: 29-30. Min.). Auch als Fernlich mehrheitlich an angloamerikanische Investoren ging, identifizierten sich Belegschaft und Betriebsräte weiterhin mit „ihrem“ Unternehmen: „Wenn man sich die Investoren anguckt, das sind alles angloamerikanische, ich glaube, das gehört zu 5\% noch Deutschen. Aber die Betriebsräte hatten 'ne unwahrscheinlich hohe Identifikation mit dem Unternehmen" (Fernlich 4. Interview: 30. Min). Für ihre Kooperationswilligkeit erwarteten die Arbeitnehmer jedoch, dass sie nicht entlassen würden, außer wenn das Unternehmen in extreme Schwierigkeiten geriet (Fernlich 2. Interview: 55. Min.). Auch dies ist eine typische Kollektivgutsituation. Die Arbeiter konnten sich nicht sicher sein, ihre Arbeitsplätze zu behalten, und das Management konnte sie nicht zwingen, sich mit dem Unternehmen zu identifizieren und motiviert zu arbeiten. Doch jede Seite vertraute auf die andere und kooperierte darum. Dies änderte sich, als Heinrich Zange den Vorstand übernahm. Im Gegensatz zu Zange habe nämlich in der Einschätzung der zuständigen Gewerkschaft „,der alte Vorstand immer noch die Mitarbeiter als ein wertvolles Gut [angesehen und war] auch aufgrund der eigenen, äh, Lebenserfahrung, eben ein anderer Managertyp [...]. Zange, man kann das so sagen, ist, 
wirklich im klassischen Sinne, von seinem gesamten Denken, eine klassische Heuschrecke, was so damit zusammenhängt" (Fernlich 4. Interview: 22-23. Min.).

Der Vorstandsvorsitzende Zange forderte nach seinem Antritt wiederholt Zugeständnisse der Arbeitnehmer und kündigte danach nichtsdestotrotz an, Produktion zu verlagern. Die Belegschaft und die Betriebsräte trugen daraufhin nicht mehr zum Kollektivgut bei, da sie meinten, dass Zange nicht mehr kooperiere. Dies war aus ihrer Sicht zwar individuell rational, jedoch kollektiv unwirtschaftlich:

Es ist heute noch so, dass Vereinbarungen mit der Geschäftsleitung ein ganz schwieriges Problem von Arbeitnehmerseite sind. Weil man sich nicht mehr traut. Dieser Konflikt hat zu einem grundsätzlich veränderten Verhalten der Betriebsräte geführt. Heute wird alles mit eigenen Sachverständigen nachgerechnet, was nicht ganz billig ist für das Unternehmen, weil es die bezahlen muss. Es wird nichts mehr geglaubt. Also, früher ging das schon mal so, da kam der Personalchef rein und dann hat man per Handschlag gesagt: ,Ok, du machst das so, ich mach das so', so diese Gentlemanagreements, die es ja in jedem Unternehmen gibt, die gibt es nicht mehr hier. Solange Zange da sein wird, wird es die nicht mehr geben. Das ist auch einer der Hauptpunkte, die aus diesem Konflikt raus gekommen sind, dass Arbeitnehmer auch richtig misstrauisch geworden sind. Die brauchen heute um was zu verhandeln dreimal so lange, wie sie vorher gebraucht haben. (Fernlich 4. Interview: 37-38. Min.)

\section{Verhaltensökonomie und Feldstudien: gemeinsame Lektionen}

Dieser Artikel hat die Frage erörtert, inwiefern die Ergebnisse verhaltensökonomischer Experimente keine Artefakte der jeweiligen Laborsituation sind, sondern sich auch in realem wirtschaftlichem Handeln widerspiegeln. Wie im Diktatorspiel geben Menschen auch in der Realität etwas ab, wenn sie dies für moralisch angemessen halten, selbst wenn dies ihren materiellen Nutzen schmälert (siehe das Unternehmen Müller). Wie im Ultimatumspiel lehnen Menschen dagegen Angebote ab, von denen sie materiell profitieren, die sie jedoch für unmoralisch halten (siehe Steche). Wie im Kollektivgutspiel kooperieren Menschen, auch wenn es individuell kurzfristig rational wäre, zu defektieren (siehe Tehnwolder). Jedoch bricht diese Kooperation zusammen, wenn ihre soziale Vorbedingung, nämlich Vertrauen, nicht mehr gegeben ist (siehe Wolder und Fernlich). Generell weisen diese Ergebnisse darauf hin, dass Menschen moralisch handeln wollen und dies je nach sozialer Situation auch tun, was wie oben beschrieben auch wirtschaftliches Handeln erklärt.

Verhaltensökonomen und auch Soziologen wird jedoch oft vorgeworfen, solche vermeintlichen „Anomalien“ wirtschaftlichen Handelns zusammenzutragen, statt empirische Ergebnisse zu einem alternativen Handlungsmodell zu bündeln (vgl. Swedberg 2005, S. 86). In diesem Sinne meint auch Diekmann (2008, S. 541): „Viele Einzelergebnisse widersprechen der ökonomischen Standardtheorie. Damit fehlt aber auch der theoretische Rahmen für die zahlreichen Befunde.“ Die Forschung zu wirtschaftlichem Handeln ist jedoch an einem Punkt angelangt, an dem sich die Kritik am Modell des Homo oeconomicus nicht mehr negativ darauf beschränken muss, es als unzutreffend darzustellen, 
sondern es kann auch ein Alternativmodell an dessen Stelle gesetzt werden, zumindest als weiter zu testende Hypothese. Ein Homo-reciprocans-Modell erklärt demnach wirtschaftliches Handeln sowohl in Experimenten als auch in Fallstudien präziser als ein Homo-oeconomicus-Modell. Denn sowohl in kontrollierten Laborexperimenten als auch in realem Unternehmerhandeln stellen Menschen ihre unmittelbaren Interessen zurück, wenn sie darauf vertrauen, dass die andere Seite dies auch tut; auch wenn dies nach einem neoklassischen Verständnis wirtschaftlichen Handelns nicht der Fall sein dürfte. Wenn die andere Seite ihre Interessen jedoch nicht zurückstellt, so vergelten Menschen ebenfalls gleiches mit gleichem. A priori gibt es keinen Grund, diesen ,shadow of indebtedness“ (Gouldner 1960, S. 174) als weniger wichtigen Aspekt menschlichen Verhaltens zu sehen als wirtschaftliche Eigennutzorientierung.

Die hier dargelegten Argumente haben einige Beschränkungen. Erstens kann an einem qualitativ rekonstruierenden Forschungsdesign kritisiert werden, dass es „so ja gar nicht war“. Auch wenn das Ziel war, möglichst ergebnisoffen an die Empirie heranzugehen, kann trotz aller methodologischer Vorkehrungen Beobachterbias nie ausgeschlossen werden. Zweitens wurde in den Felduntersuchungen immer wieder die Bedeutung von Rollen betont. Dass Steches, Wolders und Fernlichs Manager nicht auf moralische Argumente eingingen, führten auch die jeweiligen Arbeitnehmervertreter nicht darauf zurück, dass sie „schlechte Menschen“" seien. Vielmehr erklärten sie es dadurch, dass die jeweiligen Adressaten sich in einer bestimmten sozialen Rolle befanden (Manager eines Unternehmens, hinter dem Finanzinvestoren oder zerstreute Aktienhalter stehen), die es ihnen nicht erlaubte, auf moralische Appelle der Arbeitnehmer einzugehen. Umgekehrt führten Müllers oder Tehnwolders Arbeitnehmervertreter den Umstand, dass ihre Geschäftsleitung auf ihre moralischen Appelle einging, nicht darauf zurück, dass diese aus lauter Gutmenschen bestand. Vielmehr machten die Arbeitnehmervertreter dafür verantwortlich, dass ihre Geschäftsleitung, als alteingesessene Patriarchen, in der glücklichen sozialen Rolle waren, das, was ihnen als wirtschaftlich sinnvoll und moralisch geboten erschien, miteinander in Einklang bringen zu können. In diesem Sinne wären es nicht individuelle Charaktereigenschaften, wegen derer Menschen offen für moralische Appelle sind, sondern soziale Rollen.

Doch diese Erkenntnis wider den methodologischen Individualismus müsste nun umgekehrt in Experimenten geprüft werden, in denen „Rollen“, wie beispielsweise, ob jemand ein alteingesessener Familienpatriarch ist, bisher weitgehend ausgeblendet werden, auch wenn sie in der Realität menschliches Handeln nach allgemeiner Auffassung beeinflussen. Diesen Einfluss sozialer Rollen könnten Laborsettings testen, in denen Menschen dasselbe Versuchssetting vorgestellt wird, in dem sie jedoch auf unterschiedliche Rollen ,geprimed“ werden, sich also beispielsweise einmal in die Rolle eines Patriarchen und einmal in die Rolle eines Finanzinvestors hinein versetzen sollten und dann dasselbe Experiment spielen (vgl. für diese Idee auch Gintis 2010).

Eine weitere Möglichkeit, um den Einfluss von Rollen auf menschliches Verhalten zu testen, könnte darin bestehen, dieselben Menschen verschiedene Experimente durchlaufen zu lassen, in denen sie schon durch das Experiment verschiedene Rollen einnähmen. Befunde aus sozialpsychologischen Experimenten wie dem berühmten Milgram-Experiment zeigen, dass Menschen in ihrem Handeln keineswegs relativ stabil um das Wohl 
ihrer Mitmenschen besorgt sind, sondern je nach sozialer Situation anderen sogar aktiv Schaden zufügen (vgl. Milgram 1963, 1974; Burger 2009).

Mittels solch eines Variierens individueller und sozialer Faktoren, die menschliches Handeln zu beeinflussen scheinen, ist möglicherweise herauszufiltern, inwiefern moralisches Handeln überhaupt persönlichen und stabilen Charaktereigenschaften entspringt und somit relativ konstant ist oder ob es erst durch das konkrete soziale Umfeld konstituiert wird. Einstweilen muss die Frage offen bleiben, wie gesellschaftliche Rollen und persönliche Eigenschaften interagieren, um moralischen Richtlinien Geltung zu verschaffen.

Dieser Artikel versuchte demgegenüber zumindest zu zeigen, inwiefern verhaltensökonomische und soziologische Erkenntnisse durch verschiedene Methoden, jede mit ihren eigenen Schwächen, sich zu einem Handlungsmodell verdichten, welches menschliches Handeln als abhängig von der jeweiligen sozialen Situation modelliert und es damit im Experiment und im Feld besser zu erklären scheint als bisherige Annahmen.

\section{Literatur}

Aspers, Patrik. 2004. Empirical phenomenology. An approach for qualitative research. Papers in Social Research Methods. Qualitative Series No 9. London: London School of Economics and Political Science, Methodology Institute. http://www.lse.ac.uk/collections/methodologyInstitute/pdf/QualPapers/Aspers-Patrik-Phenomenology04.pdf.

Burger, Jerry M. 2009. Replicating milgram would people still obey today? American Psychologist 64:1-11.

Camerer, Colin F. 1997. Progress in behavioral game theory. The Journal of Economic Perspectives 11:167-188.

Diekmann, Andreas. 2008. Soziologie und Ökonomie: Der Beitrag experimenteller Wirtschaftsforschung zur Sozialtheorie. Kölner Zeitschrift für Soziologie und Sozialpsychologie 60:528-550.

Durkheim, Émile. 1953. The determination of moral facts (1924). In Sociology and Philosophy, Hrsg. Émile Durkheim, 35-62. Glencoe: Free Press.

Eckstein, Harry. 1975. Case studies and theory in political science. In Handbook of political science, Hrsg. Fred Greenstein und Nelson Polby, 79-138. Reading: Addison-Wesley.

Etzioni, Amitai. 1988. The moral dimension. Toward a new economics. New York: Free Press.

Etzioni, Amitai. 2003. Toward a new socio-economic paradigm. Socio-Economic Review 1:105-118.

Fehr, Ernst, und Urs Fischbacher. 2005. The economics of strong reciprocity. In Moral Sentiments and material interests. The foundations of cooperation in economic life, Hrsg. Herbert Gintis, Samuel Bowles, Robert T. Boyd, und Ernst Fehr, 151-192. Cambridge: MIT Press.

Fehr, Ernst, und Simon Gächter. 2002. Altruistic punishment in humans. Nature 415:137-140.

Fehr, Ernst, Georg Kirchsteiger, und Arno Riedl. 1993. Does fairness prevent market clearing? An experimental investigation. The Quarterly Journal of Economics 108:437-459.

Fernlich 2. Interview. (11.01.2008, 13:00-14:30).

Fernlich 4. Interview. (06.12.2007, 14:00-16:00).

Forsythe, Robert, Joel L. Horowitz, N.E. Savin, und Martin Sefton. 1994. Fairness in simple bargaining experiments. Games and Economic Behavior 6:347-369.

Freud, Sigmund. 1992. Das Ich und das Es. Bd. 11 (1923). Frankfurt a. M.: Fischer.

Friedman, Milton. 2001. The social responsibility of business is to increase its profits (1970). In Business Ethics, Hrsg. Robert Almeder, James Humber, und Milton Snoyenbos, 72-78. New York: Prometheus Books. 
George, Alexander L., und Andrew Bennett. 2005. Case studies and theory development in the social science. Cambridge: MIT Press.

Gerring, John. 2004. What is a case study and what is it good for? American Political Science Review 98:341-354.

Gintis, Herbert. 2010. Towards a renaissance of economic theory. Journal of Economic Behavior \& Organization 73:34-40.

Gintis, Herbert, Samuel Bowles, Robert T. Boyd, und Ernst Fehr. 2005. Moral sentiments and material interests: Origins, evidence, and consequences. In Moral sentiments and material interests: The foundations of cooperation in economic life, Hrsg. Herbert Gintis, Samuel Bowles, Robert T. Boyd, und Ernst Fehr, 3-39. Cambridge: MIT Press.

Glaser, Barney G., und Anselm L. Strauss. 1967. The discovery of grounded theory. Strategies for qualitative research. London: Weidenfeld and Nicolson.

Gläser, Jochen, und Grit Laudel. 2004. Experteninterviews und qualitative Inhaltsanalyse als Instrumente rekonstruierender Untersuchungen. 2.Aufl. Wiesbaden: VS Verlag für Sozialwissenschaften.

Gouldner, Alvin W. 1960. The norm of reciprocity: A preliminary statement. American Sociological Review 25:161-178.

Greiner, Ben, und Axel Ockenfels. 2009. Vom Labor ins Feld: Die Ökonomie des Vertrauens. In Wirtschaftssoziologie. 49. Sonderheft der Kölner Zeitschrift für Soziologie und Sozialpsychologie, Hrsg. Jens Beckert und Christoph Deutschmann, 219-242. Wiesbaden: VS Verlag für Sozialwissenschaften.

Güth, Werner, Rolf Schmittberger, und Bernd Schwarze. 1982. An experimental analysis of ultimatum bargaining. Journal of Economic Behavior and Organization 3:367-388.

Hall, Peter. 2007. Systematic process analysis: When and how to use it. European Political Science 7:304-317.

Healy, Kieran. 2004. Altruism as an organizational problem: The case of organ procurement. American Sociological Review 69:387-404.

Healy, Kieran. 2006. Last best gifts. Altruism and the market for human blood and organs. Chicago: University of Chicago Press.

Henrich, Joseph, Robert Boyd, Samuel Bowles, Colin Camerer, und Ernst Fehr. 2001. In search of homo economicus: Behavioral experiments in 15 small-scale societies. The American Economic Review 91:73-78.

Henrich, Joseph, Robert Boyd, Samuel Bowles, Colin Camerer, und Ernst Fehr. 2004. Introduction and guide to the volume. In Foundations of human sociality: Economic experiments and ethnographic evidence from fifteen small-scale societies, Hrsg. Joseph P. Henrich, Robert Boyd, Samuel Bowles, Colin Camerer, und Ernst Fehr, 1-7. Oxford: Oxford University Press.

Herrmann, Benedikt, Christian Thöni, und Simon Gächter. 2008. Antisocial punishment across societies. Science 319:1362-1367.

Hirschman, Albert O. 1977. The passions and the interests: Political arguments for capitalism before its triumph. Princeton: Princeton University Press.

IG Metall NRW. 2007. Interview. Düsseldorf. (04.01.2007, 10:00-12:00).

Kahan, Dan M. 2005. The logic of reciprocity: Trust, collective action, and law. In Moral sentiments and material interests. The foundations of cooperation in economic life, Hrsg. Herbert Gintis, Samuel Bowles, Robert T. Boyd, und Ernst Fehr, 339-378. Cambridge: MIT Press.

Kahneman, Daniel, Jack L. Knetsch, und Richard H. Thaler. 1986. Fairness and the assumptions of economics. The Journal of Business 59:285-300.

Lijphard, Arend. 1975. The comparable-cases strategy in comparative research. Comparative Political Studies 8:168-177.

Metall NRW. 2007. Interview. Düsseldorf. (04.04.2007, 14:00-16:30).

Milgram, Stanley. 1963. Behavioral study of obedience. Journal of Abnormal and Social Psycho$\log y$ 67:371-378. 
Milgram, Stanley. 1974. Obedience to authority. An experimental view. New York: Harper.

Müller. 1. Interview. (02.05.2007, 15:30-16:30).

Müller. 2. Interview. (08.02.2007, 12:00-14:00).

Ostrom, Elinor. 1990. Governing the commons: The evolution of institutions for collective action, the political economy of institutions and decisions. Cambridge: Cambridge University Press.

Ostrom, Elinor. 2005. Policies that crowd out reciprocity and collective action. In Moral sentiments and material interests. The foundations of cooperation in economic life, Hrsg. Herbert Gintis, Samuel Bowles, Robert T. Boyd, und Ernst Fehr, 253-275. Cambridge: MIT Press.

Parsons, Talcott. 1937. The structure of social action. New York: Free Press.

Polanyi, Karl. 1944. The great transformation. New York: Farrar \& Rinehart.

Schütz, Alfred. 1932. Der sinnhafte Aufbau der sozialen Welt. Eine Einleitung in die verstehende Soziologie. Wien: Springer.

Schütz, Alfred. 1971. Gesammelte Aufsätze I. Das Problem der sozialen Wirklichkeit. Den Haag: Nijhoff.

Steche 1. Interview. (24.01.2008, 10:00-11:00).

Steche 2. Interview. (16.05.2008, 9:00-11:00).

Steche 3. Interview. (08.10.2007, 14:00-17:00).

Strauss, Anselm L., und Juliet Corbin. 1990. Basics of qualitative research. Grounded theory procedures and techniques. Newbury Park: Sage.

Streeck, Wolfgang. 2010. Does 'Behavioural Economics' offer an alternative to the neoclassical paradigm? Socio-Economic Review 8:387-397.

Swedberg, Richard. 2005. Interest. New York: Open University Press.

Tehnwolder 1. Interview. (20.05.2008, 12:30-14:00).

Tehnwolder 2. Interview. (25.01.2008, 14:00-15:30).

Thaler, Richard H. 1992. The winner's curse. Paradoxes and anomalies of economic life. Princeton: Princeton University Press.

Titmuss, Richard M. 1971. The gift relationship. From human blood to social policy. New York: Pantheon Books.

Weber, Max. 1978. Gesammelte Aufsätze zur Religionssoziologie (1920). 7.Aufl. Tübingen: J.C.B. Mohr.

Weber, Max. 1988. Gesammelte Aufsätze zur Religionssoziologie I (1920). 9.Aufl. Tübingen: UTB.

Williamson, Oliver. 1985. The economic institutions of capitalism. New York: Free Press.

Wittgenstein, Ludwig. 1953. Philosophical investigations. Oxford: Blackwell.

Wolder 1. Interview. (04.04.2008, 11:00-13:00).

Wolder 2. Interview. (06.05.2008, 13:30-17:00).

Wolder/Tehnwolder 1. Interview. (10.01.2008, 11:45-13:00).

Wolder/Tehnwolder 2. Interview. (10.01.2008, 9:00-10:30).

Wrong, Dennis. 1961. The oversocialized conception of man in modern sociology. American Sociological Review 26:183-193.

Zelizer, Viviana. 1978. Human values and the market: The case of life insurance and death in 19th century America. American Journal of Sociology 84:591-610.

Zelizer, Viviana. 1979. Morals and markets: The development of life insurance in the United States. New York: Columbia University Press.

Zelizer, Viviana. 1981. The price and value of children: The case of children's insurance in the United States. American Journal of Sociology 86:1036-1056.

Zelizer, Viviana. 1985. Pricing the priceless child: The changing social value of children. New York: Basic Books.

Zelizer, Viviana. 2007. Ethics in the economy. Zeitschrift für Wirtschafts- und Unternehmensethik $8: 8-23$. 
Martin Schröder, 1981, Postdoc-Stipendiat am Max-Planck-Institut für Gesellschaftsforschung, Köln. Forschungsgebiete: Moral und wirtschaftliches Handeln, Wirtschaftssoziologie, vergleichende Politische Ökonomie. Veröffentlichungen: Regional and Sectoral Varieties of Capitalism, Economy and Society, 38, 2009 (mit C. Crouch und H. Voelzkow); Integrating Welfare and Production Typologies. How Refinements of the Varieties of Capitalism Approach Call for a Combination with Welfare Typologies, Journal of Social Policy 38, 2009; Wie moralische Argumente wirtschaftliches Handeln beeinflussen. Frankfurt a. M./New York (in Vorbereitung). 